\title{
The Art of EMS
}

\author{
10th Anniversary \\ EMS Today \\ Conference \& Exposition
}

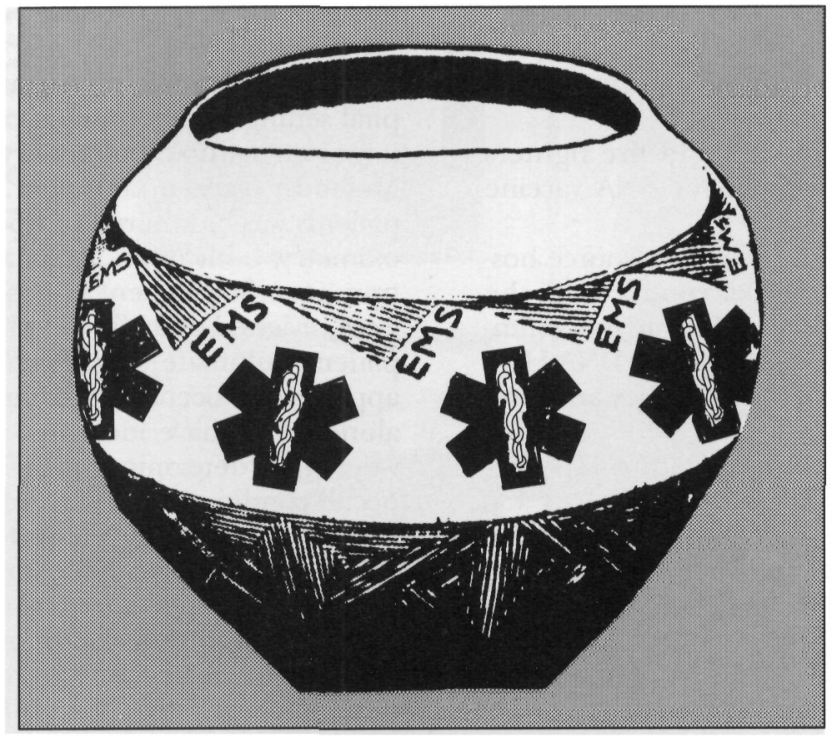

March 11-14, 1992

Albuquerque, New Mexico

Plan now to join 1500 of the

brightest and most influential EMS professionals

for the biggest and most celebrated

EMS educational event of 1992.

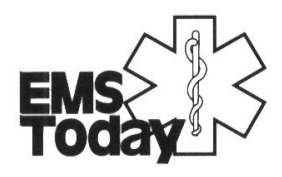

Sponsored by

JEMS Communications and The Conference Corporation

P.O. Box 2400, Carlsbad, CA 92018-2400

$619 / 431-8088$ 


\section{Scientific Oral Paper Presentations}

\author{
Track I \\ Great Hall Center
}

1:00 PM

Seroconversion of HBV-DNA Vaccinated EMS Personnel

Koenigsberg MS, Ward S, Koslenko T, Herman L,

McDonald C, Feigon KL, Feldman RM

University of Illinois College of Medicine, Chicago, Illinois; Chicago

North EMS System, Illinois Masonic Medical Center, Chicago, Illinois

Objective: To evaluate seroconversion rate of EMS personnel given the HBV recombinant DNA vaccine Series.

Design: Retrospective review of paramedics and fire fighters consecutively completing the $\mathrm{HBV}$ recombinant DNA vaccine program.

Setting: Urban, Non-profit, private, regional EMS resource hospital, the Chicago North EMS System is under contract with the Chicago Fire Department. This contract allows for the voluntary vaccination of EMS personnel with recombinant DNA-HBV vaccine and treatment and follow-up of paramedics and firemen possible exposed to HBV.

Population: Initial 50 EMS personnel completing HBV recombinant $\mathrm{DNA}$ vaccine program.

Intervention: The HBV recombinant DNA series was voluntarily administered per manufacturer's recommendations to Chicago North EMS System paramedics and firemen. After two weeks post-vaccination series completion, HBV antibody titres were drawn to determine reactivity and need for further immunization.

Results: Of the $\mathbf{5 0}$ vaccinated individuals, only two were found to have negative titre $(4 \%)$. These two individuals were given a fourth dose of vaccine with successful seroconversion.

Conclusions: Prehospital care personnel are known, high-risk, health care workers for acquiring $\mathrm{HBV}$ and should be prophylactically vaccinated. there is a high seroconversion rate $(96 \%)$ utilizing $\mathrm{HBV}$ recombinant DNA vaccine, however, post-vaccination antibody titres must be performed to identify those rare individuals that do not seroconvert.
Track I

Great Hall Center

Friday, June 14, 1991

Moderator: Donald Yealy

1:15 PM

The Use of Pulse Oximetry in the

Prehospital Detection of Occult Disease

Fugelso DK, Saunders CE, Knudson MM

University of California, San Francisco, School of Medicine; San

Francisco Department of Public Health, San Francisco, Califormia

Purpose: This study investigated pulse oximetry in the prehospital setting to determine its value in detecting occult clinically important cardiac, pulmonary, or hypoxic conditions.

Methods: Oxygen saturation $\left(\mathrm{SO}_{2}\right)$ in critically ill, prehospital patients was monitored prospectively by paramedics using pulse oximetry, both upon initial arrival and during treatment and transport. Subsequent in-hospital clinical and laboratory data were reviewed by a blinded physician reviewer, to determine the patient's ultimate diagnosis. The diagnosis was categorized as apparent or occult based on whether prehospital assessment alone would have identified the condition. Cutoff $\mathrm{SO}_{2}$ values were then determined which would have detected clinically occult conditions with the greatest sensitivity (SENS), specificity (SPEC), positive (PPV), and negative (NPV) predictive value (test of proportions: chi-square analysis).

Results: A total of 201 patients were studied; 39 (19.4\%) had clinically important conditions of which $32(82.1 \%)$ were occult. On room air, 12 of 18 occult conditions would have been detected using $\mathrm{SO}_{2}$ values less than $92 \%$ ( $\mathrm{p}=.0001$ ); SENS $66.7 \%$, SPEC 91.3, PPV 57.1, NPV 94.0); in patients on supplemental oxygen, nine of 14 would have been detected using SO2 values less than $98 \%$ (p-.01; SENS 64.3, SPEC 69.8, PPV 36.0, NPV 88.1).

Conclusions: Pulse oximetry is a useful, non-invasive technology in the prehospital setting which aids the early detection of clinically important occult injury or illness. 

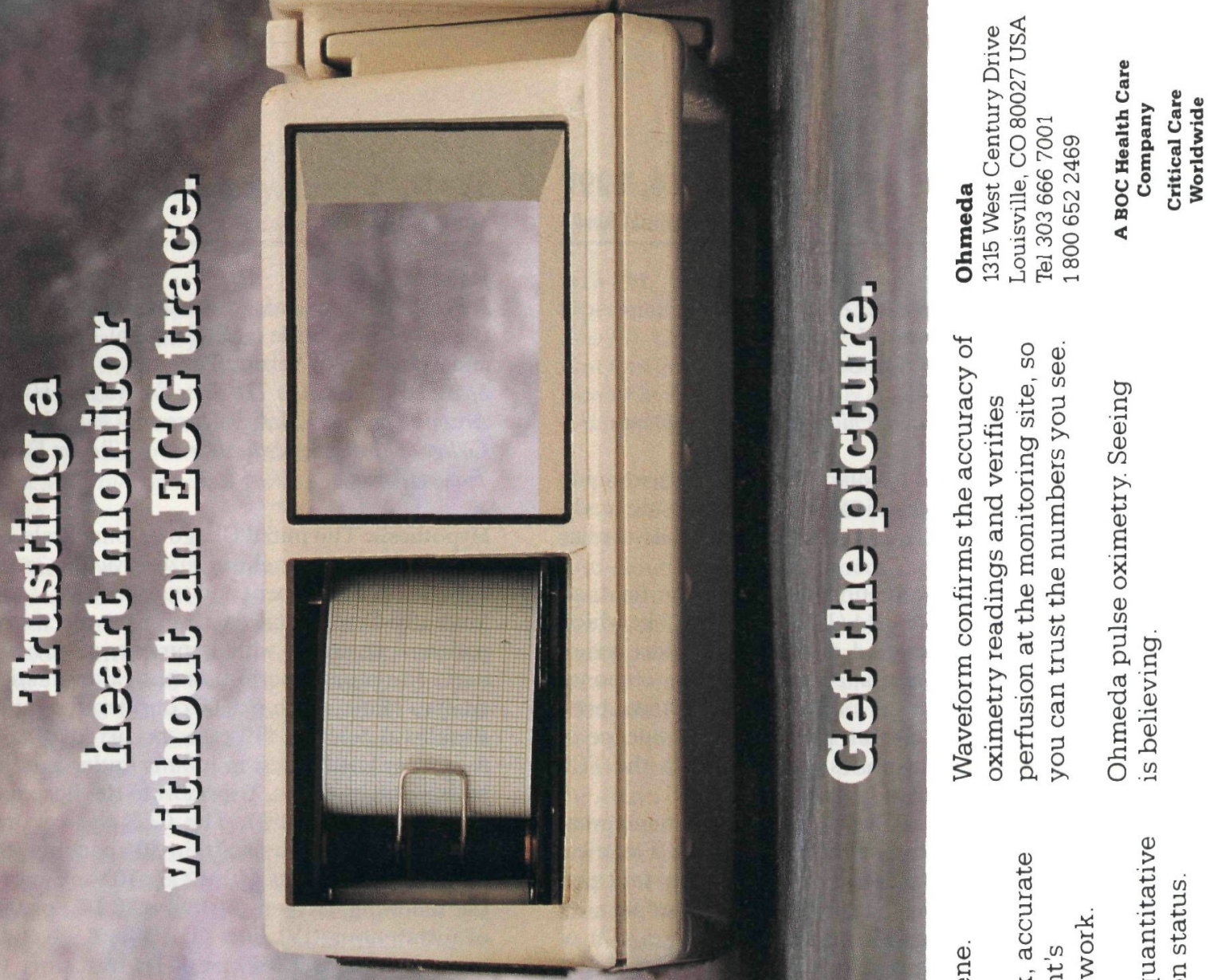

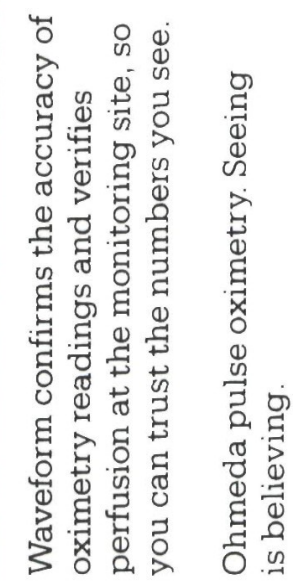

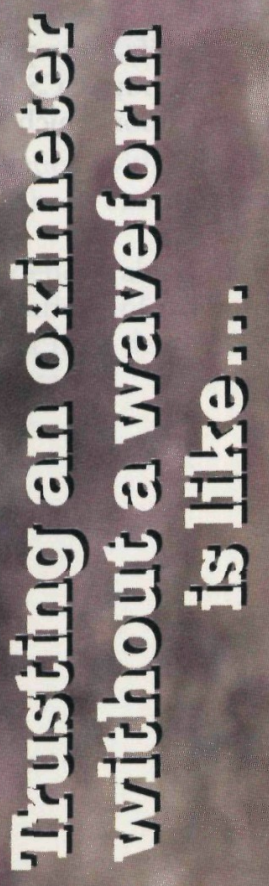

S

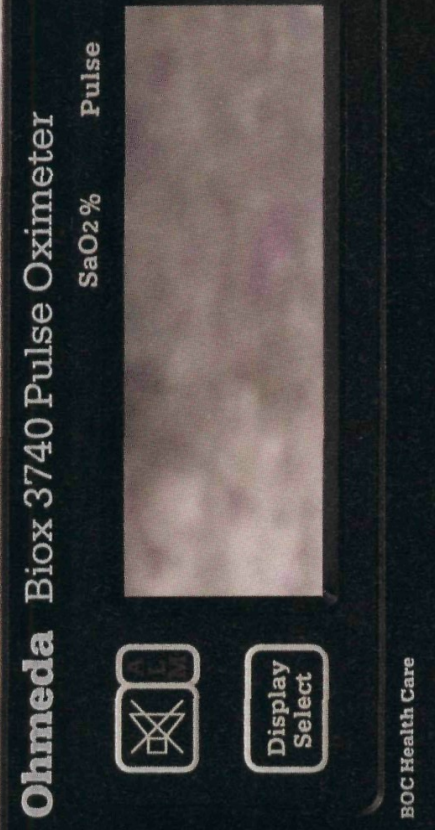

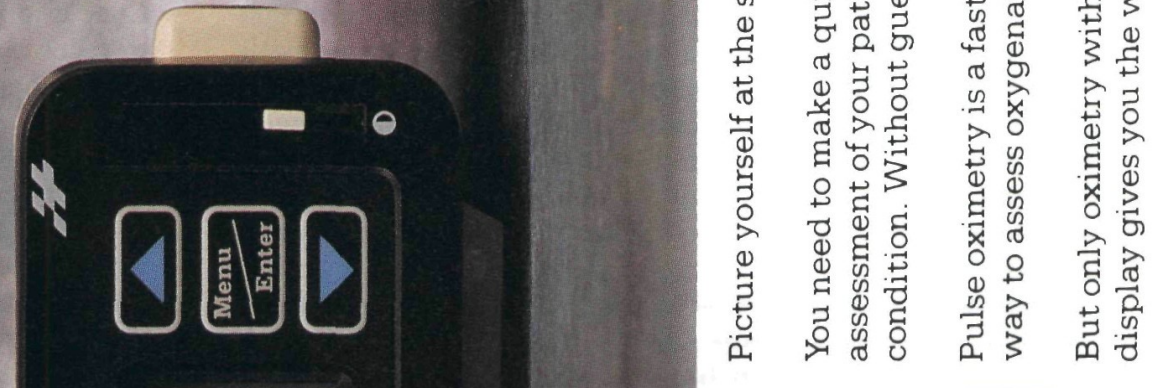
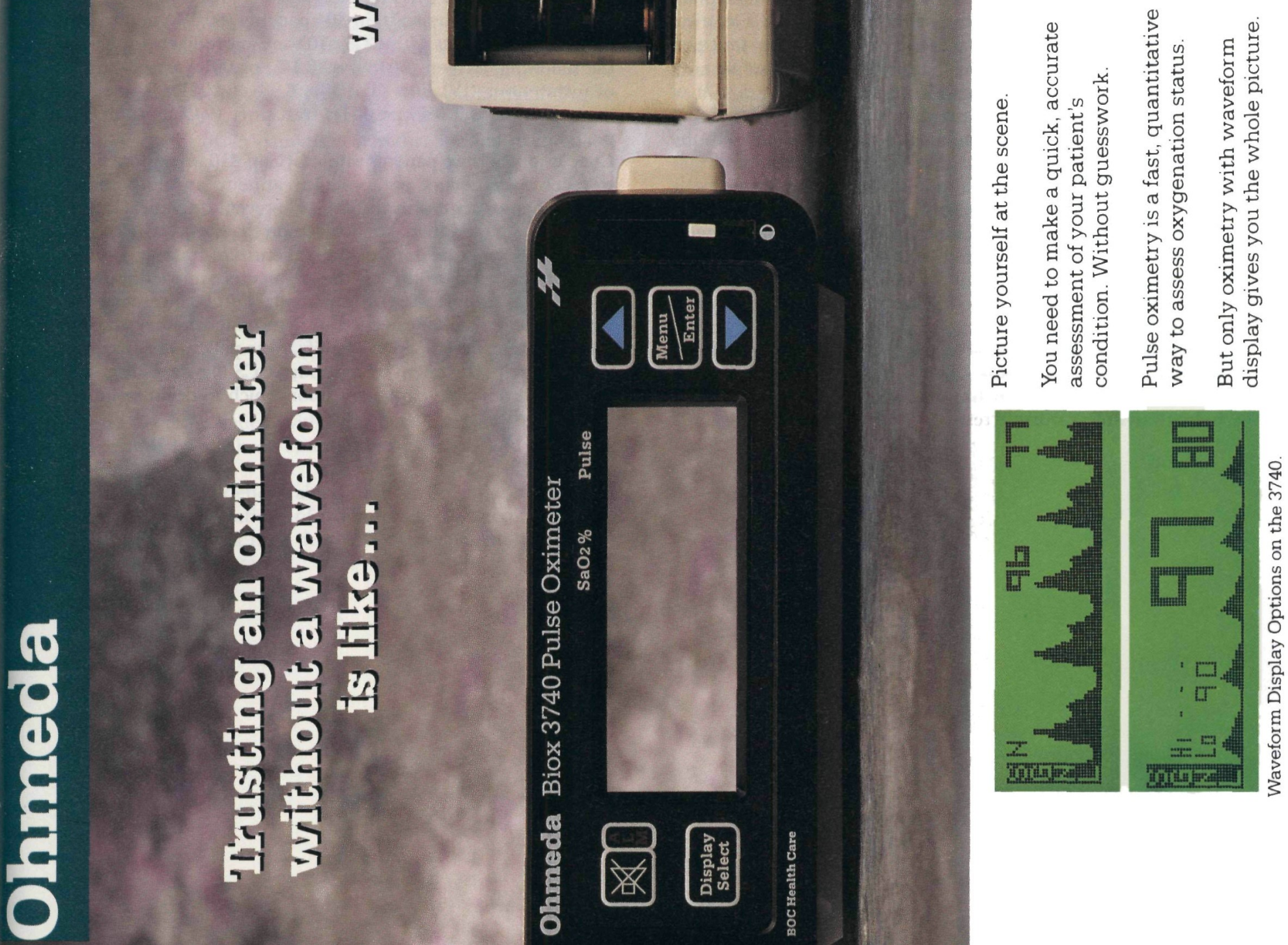


\section{Track I}

Great Hall Center

\section{1:30 PM}

Results of Transcutaneous Cardiac

Pacing in an Urban EMS System

Cionni DJ, Spillane LL, Angelini KM, Cohen SB,

Klein SW, Lindell DW, Davidson SJ

The Medical College of Pennsylvania, Philadelphia, Pennsylvania

Hypothesis: Transcutaneous cardiac pacing (TCP) may improve perfusion in prehospital patients with symptomatic bradycardias and restore perfusion to those in bradyasystolic cardiac arrest.

Methods: Following the introduction of TCP to the field, all pre-hospital patient encounters $(4 / 1 / 90-2 / 14 / 91)$ in which TCP was attempted were identified during $100 \%$ chart review of ALS. Available data were evaluated to obtain the response time, duration of pulselessness if known, initial rhythm, presence of pulses prior to TCP, rhythm prior to TCP, evidence of electrical capture, presence of pulses consistent with the TCP rate and administration of ACLS drugs.

Results: TCP was attempted in 78 patients. Four patients were excluded from analysis because of incomplete data. Electrical capture was noted in $64 \%(47 / 74)$ of patients. Ninety-four percent $(44 / 47)$ of these patients had received atropine without improvement prior to pacing. Fifty-three percent $(25 / 47)$ of these patients had no pulses prior to pacing and $56 \%(14 / 25)$ of these patients developed pulses at a rate consistent with the TCP rate. Forty-seven percent $(22 / 47)$ of patients with electrical capture had pulses prior to TCP but had a symptomatic bradycardia. Ninety-five percent $(21 / 22)$ of these patients developed pulses consistent with the TCP rate. Two of these 22 patients had been resuscitated from a pulseless rhythm to a symptomatic bradycardia. Thus, a total of $74 \%(35 / 47)$ of patients with electrical capture developed pulses consistent with the TCP rate.

Conclusions: TCP applied according to standing orders in an urban EMS system is capable of improving perfusion in patients with symptomatic bradycardias and restoring perfusion to those in bradyasystolic cardiac arrest.
Track I

Great Hall Center

Friday, June 14, 1991

Moderator: Donald Yealy

1:45 PM

A Prospective Analysis of the Impact of Initial

Glasgow Coma Scale on Prehospital Treatment

and Transport of Seizure Patients

Spaite DW, Valenzuela TD, Criss EA, Meislin HW, Ross J

Arizona Emergency Medicine Research Center, University of Arizona

College of Medicine; Medical Division, Tucson Fire Department,

Tucson, Arizona

Hypothesis: The initial Glasgow Coma Scale (GCS) obtained by prehospital personnel on seizure victims is associated with the likelihood of treatment and transport.

Methods: Prehospital data were prospectively collected for all patients presenting with seizures to an urban EMS system during a five-month period. Statistical evaluation was chi-square analysis (Yates') with $\mathrm{p}<0.05$ considered significant.

Results: A total of 419 cases occurred (62.8\% male). Seizure frequency was highest in infants under age three and in adults in their late twenties. Transport to the hospital was arranged for 289 patients $(69.0 \%)$. The GCS was recorded in 378 cases $(90.2 \%)$. The GCS was $>10$ in 304 patients $(80.4 \%)$ and $\leq 10$ in $74(19.6 \%)$. Patients with GCS $\leq 10$ were more likely to receive the following: oxygen ( $50.0 \%$ vs $20.1 \%, \mathrm{p}<.001)$; establishment of intravenous (IV) line $(35.1 \%$ vs $8.9 \%, \mathrm{p}<.001)$; IV medications $(16.2 \%$ vs $1.0 \%, \mathrm{p}<.001)$; transport $(94.6 \%$ vs $63.8 \%$, $\mathrm{p}<.01)$.

Conclusion: Seizure patients with a GCS of $\leq 10$ were more likely to receive a variety of prehospital treatments and to be transported than patients with GCS $>10$. However, the clinical indicators that were used to make the decision that it was "safe" not to transport nearly one-third of the patients are unclear. Essentially, no data exist regarding the parameters impacting treatment and transport of seizure patients. Future investigations, with outcome data, are needed to determine whether low risk criteria can be developed to identify those patients (if any) that do not require treatment or transport. GCS may provide an objective, reproducible parameter upon which to begin formulating such criteria. 
Track I

Great Hall Center

Friday, June 14, 1991

Moderator: Donald Yealy

\section{2:00 PM}

Emergency Physician Interpretation of Prehospital,

Paramedic-Approved Electrocardiograms

Schaffer JA, Valenzuela TD, Wright AL, Clark L,

Brakema R, Goldman S, Spaite DW

Arizona Emergency Medicine Research Center. Department of

Mathematics, University of Arizona; Section of Cardiology,

Department of Internal Medicine, Veterans Administration Medical

Center, Tucson, Arizona

Hypothesis: Emergency Physician interpretation of prehospital, paramedic-acquired electrocardiograms (ECGS) is accurate judged by comparison with that of a reference cardiologist.

Methods: Over a two-month period, 12 lead ECGs were obtained by paramedics in the field from 73 patients presenting with acute chest pain. The ECGs were transmitted by cellular telephone to a central location. Each ECG was assessed for the presence of acute myocardial infarction (AMI) by a third-year, emergency medical resident (EMP-R), a residency-trained, board-certified, emergency physician (EMP-RT), an emergency physician, board certified under practice option (EMP-PT), and a board-certified cardiologist. Agreement between each emergency physician and the cardiologist was determined by the kappa statistic. Hospital records were reviewed for final diagnosis of each patient.

Results: Eight of 75 patients received a hospital diagnosis of AMI. See Table 1.

Table 1-Results of Physician Interpretation of ParamedicAcquired ECGs.

$$
\text { Total \% }
$$

\begin{tabular}{|c|c|c|c|c|}
\hline \multicolumn{2}{|c|}{ Agreement } & kappa & Sensitivity & Specificity \\
\hline EMPR & 97 & .625 & .125 & .969 \\
\hline EMP-RT & 99 & .920 & .250 & .969 \\
\hline EMP-PT & 97 & .625 & .125 & .969 \\
\hline Cardiologist & NA & NA & .250 & .985 \\
\hline
\end{tabular}

The ECG of one patient with Prinzmetal's angina was interpreted as AMI by all three emergency physicians and the cardiologist.

Conclusions: Residency-trained or board-certified emergency physician interpretations of prehospital, paramedic-acquired, 12-lead ECGs who a high degree of agreement with reference cardiologist interpretation.
Track I

Friday, June 14, 1991

Great Hall Center

Moderator: Donald Yealy

2:15 PM

Operational Safety on EMS Helicopters: A Longitudinal Study

Low RB, Carney M, Collins P, Defesne D, Hunt RC

East Carolina University, Pitt County Memorial Hospital, Greenville,

North Carolina; The University of Chicago Aeromedical Network,

Chicago, Illinois

Hypothesis: Emergency medical service (EMS) helicopter accidents are a concern of EMS personnel and the public. This research tests the hypotheses that: 1) helicopters permitted to fly into clouds under instrument flight rules (IFR) have a better operational safety record than helicopters limited to flight under visual flight rules; 2) programs are shifting to the use of helicopters certified for flight under IFR; and 3) busy programs have fewer accidents than programs that fly less frequently.

Methods: This is a longitudinal, epidemiologic study of all helicopter ambulance services in the United States and Canada. Four surveys cover the period between 1 January 1986-28 February 1991. Data were collected on the number of safely completed flights and the number of accidents. Statistical comparisons were done with chi-square and log likelihood ratio chisquare tests.

Results: Response rate for the first surveys was $98 \%$; a similar response rate was expected from the last survey. Helicopter programs that can always fly under IFR had no accidents. Helicopter programs that are sometimes or always limited to visual flight had an accident rate of $58 /$ million flights $(p=.033)$. The percentage of patients flown on IFR certified helicopters has increased from $16 \%$ to $29 \%$ ( $p<.0005)$. Programs which fly frequently have a better safety record ( 24 accidents/million flights) than programs that fly infrequently (131 accidents/million flights, $\mathrm{p}<.001$ ).

Conclusions: The ability to fly "on instruments" seems to improve the operational safety of civilian helicopter ambulances. Programs are shifting to the use of these more expensive aircraft. Busy flight programs have a significantly better safety record than programs that less frequently. 

Track I

Friday, June 14, 1991

Great Hall Center

\section{2:30 PM}

United States Trauma Diversion

Policy and Triage Criteria Utilization

Kolka J, Sloan E, Koenigsberg M, Nolin J, Fagan J,

Stein-Spencer L, Barrett J

Department of Emergency Medicine and Division of Trauma, Cook County Hospital; Department of Emergency Medicine, University of

Illinois Hospital; Illinois Department of Health; Program in

Emergency Medicine, University of Illinois College of Medicine,

Chicago, Illinois

Purpose: Identification of criteria used by emergency medical service (EMS) systems in the United States to divert trauma patients to specialty care.

Study Design: This study conducted a cross-sectional mail and telephone $\mathrm{f}$, follow-up survey of state and local EMS officials addressing trauma triage in the largest EMS system in each state.

Results: Surveys were returned from 40 states (80\%). A formal state or local system which allowed diversion to trauma centers was present in 24 of 30 states $(60 \%)$, of which 22 had written documents governing trauma diversion (92\%). Eligibility for diversion was determined solely by paramedics in 14 systems $(59 \%)$, medical control in five systems $(21 \%)$, and jointly in five systems (20\%). Seventeen of $24(70 \%)$. included "relative or judgment criteria." Formal, published, decision schemes were incorporated into the 17 systems (70\%). Eight systems $(33 \%)$ used the American College of Surgeons decision scheme exclusively. The most commonly used physiologic criteria was low systolic blood pressure, which was used in 19 systems (79\%), followed by respiratory abnormalities, used by 14 systems $(58 \%)$. Traumatic arrest, alone, causes diversion in 12 systems $(50 \%)$. Penetrating head trauma, alone, causes bypass in 16 systems $(66 \%)$. Although the presence of flail chest prompted diversion in 15 systems $(62.5 \%)$, other blunt injuries were absolute indications for diversion in only seven systems $(29 \%)$.

Conclusion: A large number os states have no formal trauma network within EMS systems that allow for triage to trauma centers. In systems which allow for diversion, nearly all have policies which include specific criteria. The majority allow paramedic judgment to prompt diversion even when specific criteria are not met. the most frequently used criteria are anatomic (penetrating) injuries, followed by low systolic blood pressure. Formal (published) decision schemes were used to some extent in 17 systems $(70 \%)$

Track II

Great Hall Center

Friday, June 14, 1991

Moderator: Daniel Spaite

\section{3:30 PM}

Infant Ventilation and Oxygenation by Basic

Life Support Providers: Comparison of Methods

Terndrup TE, Warner DA, Pierluisi GJ

Department of Emergency Medicine and Pediatrics, The State

University of New York Health Science Center at Syracuse; the School

of Medicine, The State University of New York. Health Science Center

at Syracuse, Syracuse, New York

Little information is available on the performance of basic life support (BLS) providers in infant ventilation. The hypothesis of this study is that there are no significant differences between mouth-to-mouth (M-M), mouth-to-mask (M-Ma), and pediatric $(\mathrm{PBM})$, or adult (ABM) bag-mask methods in the percentage of acceptable breaths when performed by BLS personnel. Fifty, certified, BLS providers performed five ventilation methods in random sequence for 60 seconds each on a $5 \mathrm{~kg}$ infant mannequin following standardized instructions. Supplemental oxygen, $10 \mathrm{~L} / \mathrm{min}$ was supplied with one M-Ma trial and PBM methods. Airway patency, peak airway pressure (PAP), respiratory rate, tidal volume, and delivered oxygen concentration $\left(\mathrm{FiO}_{2}\right)$ were recorded. The percentage of breaths with excessive PAP (i.e., $30 \mathrm{mmHg}$ ), percentage of acceptable breaths using loose (i.e., $25-125 \mathrm{ml}$ ) and strict (i.e., $50-100 \mathrm{ml}$ ) criteria, and $\mathrm{FiO}_{2}$ at $15 \mathrm{~s}, 30 \mathrm{~s}, 45 \mathrm{~s}$, and $60 \mathrm{~s}$ were compared among ventilation methods using ANOVA. For all subjects and those with a patent airway ( $n=36$ ), there were no significant differences in the percentage of acceptable breaths among the ventilation methods using loose or strict criteria. The percentage excessive pressure breaths for PBM (56 \pm 6.0 ; mean \pm SEM; all subjects) and ABM

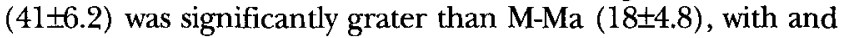
without a patent airway. Although $\mathrm{RR}$ and percentage excessive pressure breaths were not significantly different, the percentage acceptable breaths and $\mathrm{FiO}_{2}$ delivery with each ventilation method was significantly better in the patent airway group. In BLS providers, M-Ma ventilation with supplemental oxygen provided the best method of initial infant ventilation based upon the percent of acceptable breaths, oxygen delivery, and fewest excessive pressure breaths. 


\section{Track II}

Great Hall Center
Friday, June 14, 1991

Moderator: Daniel Spaite

\section{3:45 PM}

Demand Valve Ventilation in a Swine Pneumothorax Model

Murray J, Menegazzi J, McCabe J, Seaberg $D$

University of Pittsburgh Affiliated Residency in Emergency Medicine,

Division of Emergency Medicine, University of Pittsburgh Center

for Emergency Medicine of Western Pennsylvania, Pittsburgh,

Pennsylvania

Hypothesis: Bag valve mask (BVM) and oxygen-powered demand valves (OPDV) are two available adjuncts for artificial ventilation. Use of OPDVs have been limited by concern for causing or worsening pneumothorax (PTX). The hypothesis of this investigation was that the OPDV and the BVM do not differ in their effects on PTX volume, and hemodynamic or ventilatory parameters.

Methods: In this randomized, cross-over design, five fasted female swine $(16-23 \mathrm{~kg}$, mean $20.5 \mathrm{~kg}$ ) were sedated (ketamine/xylazine), anesthetized (alpha chloralose), intubated with a 5-0 cuffed endotracheal tube, ventilated with room air, paralyzed (vecuronium), and left femoral arterial lines placed. Right chest injury was induced using a 14-gauge ( $\mathrm{g}$ ) needle to produce disruption of underlying lung parenchyma. An $18 \mathrm{~g}$ French Foley catheter was inserted and sealed in the right pleural space at the injury site. For each trial, a PTX was induced by instilling 300 cc of air,. Each subject underwent 10-minute ventilation trials (to simulate prehospital transport times) with OPDV and with BVM by the same investigator, blinded to instrumentation. Measurements of post-trial PTX volume, heart rte (HR), blood pressure (BP), end-tidal $\mathrm{CO}_{2}\left(\mathrm{ETCO}_{2}\right)$, and arterial blood gases (ABGs) were made. Data were compared using repeated measures ANOVA, Tukey's multiple comparisons, and Student's $t$ test with the alpha error rate set at 0.05 .

Results: There was no significant difference in PTX volume after OPDV ventilation $(310.8 \mathrm{cc})$ versus $B V M$ ventilation $(315.4 c c)(p=821)$. No significant differences in HR, BP, or ABGs were detected. The $\mathrm{ETCO}_{2}$ showed significant decreases from baseline in both OPDV (33.0 to 28.6) and BVM (33.8 to 30.2 ) trials. However, multiple comparisons showed no significant differences between OPVD and BVM at any timepoints.

Conclusion: In this model, OPDV and BVM ventilation did not differ in their effects on PTX volume or hemodynamic variables. No animal showed signs of tension PTX. Hyperventilation occurred with both devices, but this may have been operator-dependent.

Track II

Great Hall Center

Friday, June 14, 1991

Moderator: Daniel Spaite

\section{4:00PM}

Comparison of Delivered Volumes and Airway Pressures

when Ventilating through an Endotracheal Tube with

a Bag-Valve Demand Valve

Mossesso V, Lukitsch K, Menegazzi J, Mossesso J

University of Pittsburgh Affiliated Residency in Emergency Medicine,

University of Pittsburgh Medical Center, Center for Emergency

Medicine of Western Pennsylvania, Pittsburgh, Pennsylvania

Purpose: Use of an oxygen-powered demand valve (DV) to ventilate through an endotracheal tube is generally considered inappropriate. This study hypothesized that ventilation with a bag-valve device (BV) and the recently re-designed DV would produce similar tidal volumes (TV), minute ventilation, and peak airway pressures (PAP).

Methods: This is a prospective, randomized, in vitro, experimental model. Subjects were blinded to volume and pressure gauges. Thirty-nine emergency medical technicians (EMTs) (mean age 27 years; mean experience 5 years) volunteered to ventilate a mechanical test lung through an endotracheal tube for ten minutes. Each subject was randomized to $\mathrm{BV}$ and to either normal $\left(0.05 \mathrm{~L} / \mathrm{cm} \mathrm{H}_{2} \mathrm{O}\right)$ or poor $\left(0.02 \mathrm{~L} / \mathrm{cm} \mathrm{H}_{2} \mathrm{O}\right)$ lung compliance. This DV delivers set flow of $40 \mathrm{~L} / \mathrm{min}$ at a maximum $50 \pm 5 \mathrm{~cm} \mathrm{H}_{2} \mathrm{O}$. Subjects were instructed to use their "usual" technique for an average size adult in respiratory arrest with normal HR and BP. The TV and PAP were recorded for each breath; the MV and maximum PAP (PAP ${ }_{\max }$ ) for each minute was noted. Data were analyzed using repeated measures, ANOVA, and Turkey multiple comparisons with alpha set at 0.05 .

Results:

\begin{tabular}{|c|c|c|c|}
\hline \multirow[b]{2}{*}{ MINUTE } & \multicolumn{3}{|c|}{ TV (ml) } \\
\hline & 1 & 5 & 10 \\
\hline $\mathrm{BV}$ & $1079(189)^{*}$ & $1089(134)^{*}$ & $1120(111)^{*}$ \\
\hline \multirow[t]{2}{*}{ DV } & $899(477)$ & $902(582)$ & $949(550)$ \\
\hline & \multicolumn{3}{|c|}{ MV (L) } \\
\hline MINUTE & 1 & 5 & 10 \\
\hline BV & $18.4(6.0)$ & $18.8(5.6)$ & $18.2(6.0)$ \\
\hline \multirow[t]{2}{*}{ DV } & $14.0(6.7)$ & $14.9(7.3)$ & $15.7(7.6)$ \\
\hline & \multicolumn{3}{|c|}{$\operatorname{PAP}\left(\mathrm{cm} \mathrm{H}_{2} \mathrm{O}\right)$} \\
\hline MINUTE & 1 & 5 & 10 \\
\hline BV & $23(6)^{*}$ & $27(5) *$ & $27(6)^{*}$ \\
\hline \multirow[t]{2}{*}{$\mathrm{DV}$} & $10(4)$ & $10(4)$ & $11(5)$ \\
\hline & \multicolumn{3}{|c|}{$\mathrm{PAP}_{\operatorname{mix}}\left(\mathrm{cm} \mathrm{H}_{2} \mathrm{O}\right)$} \\
\hline MINUTE & 1 & 5 & 10 \\
\hline BV & $26(7)^{*}$ & $29(6)^{*}$ & $31(8)^{*}$ \\
\hline DV & $12(4)$ & $12(5)$ & $13(6)$ \\
\hline
\end{tabular}

\begin{tabular}{|c|c|c|c|}
\hline \multicolumn{4}{|c|}{ Poor Compliance $\left(0.02 \mathrm{~L} / \mathrm{cm} \mathrm{H}_{2} \mathrm{O}\right)$, Mcan (SD } \\
\hline \multirow[b]{2}{*}{ MINUTE } & \multicolumn{3}{|c|}{ TV (ml) } \\
\hline & 1 & 5 & 10 \\
\hline BV & $1059(152)$ & 1 (10992(161) & $1070(170)$ \\
\hline DV & $1083(991)$ & $1102(412)$ & $1104(829)$ \\
\hline \multirow[b]{2}{*}{ MINUTE } & \multicolumn{3}{|c|}{ MV (L) } \\
\hline & 1 & 5 & 10 \\
\hline BV & $14.8(3.7)$ & $15.3(4.8)$ & $\mid 6.0(4.8\}$ \\
\hline DV & $15.6(6.4)$ & $15.8(6.3)$ & $15.9(5.6)$ \\
\hline \multirow[b]{2}{*}{ MINUTE } & \multicolumn{3}{|c|}{$\mathrm{PAP}\left(\mathrm{cm} \mathrm{H}_{2} \mathrm{O}\right)$} \\
\hline & 1 & 5 & 10 \\
\hline BV & $32(7)^{*}$ & $38(8) *$ & $39(9)^{*}$ \\
\hline DV & $22(7)$ & $22(8)$ & $23(6)$ \\
\hline \multirow[b]{2}{*}{ MINUTE } & \multicolumn{3}{|c|}{ 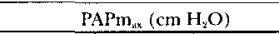 } \\
\hline & 1 & 5 & 10 \\
\hline BV & $36(8)^{*}$ & $42(7)^{*}$ & $44(7)^{*}$ \\
\hline DV & $25(8)$ & $26(8)$ & $25(7)$ \\
\hline
\end{tabular}

${ }^{*}$ p $<$.01, significantly higher than DV by ANOVA and Turkey multiple comparisons,

Conclusions: In this model, ventilation with BV and DV both provided more than adequate $\mathrm{TV}$ and MV; values were similar except for higher TV with BV at normal compliance However, DV yielded significantly lower PAPs and PAP $_{\text {max }}$ than BV over each minute at both poor and normal compliance. These findings need corroboration in an in vivo model, but suggest that with proper training DV ventilation through an endotracheal tube may be advantagous. 



\section{Track II}

Great Hall Center
Friday, June 14, 1991

Moderator: Daniel Spaite

\section{4:15 PM}

Confirmation of Prehospital Endotracheal Intubation Using an Infrared End-Tidal $\mathrm{CO}_{2}$ Detector McCabe L, Heller MB, Doctor A

University of Pittsburgh Affiliated Residency Emergency Medicine, The University Pittsburgh School of Medicine, and the Center for Emergency Medicine of Western Pennsylvania, Pittsburgh, Pennsylvania

Hypothesis: A miniature, infrared, solid-state, end-tidal $\mathrm{CO}_{2}$ detector (MSA Minicap III) can quickly and accurately confirm endotracheal tube placement in the prehospital setting, especially during cardiac arrest.

Methods: A total of 25 consecutive, adult patients requiring endotracheal intubation in the field were prospectively entered into the study. Intubation was performed by paramedics or emergency medicine physicians from the City of Pittsburgh. Difficulty of intubation as well as confirmation was recorded in a scale of zero to three, zero representing no difficulty and three representing most difficult. Intubation was confirmed by CXR or visualization. Time without a perfusing rhythm before intubation also was recorded. Twenty-five randomly selected historical controls were compared for age, indication for intubation, and mean duration of absent perfusion using Fisher's Exact Test.

Results: Tracheal intubation was achieved by the orotracheal route in 23 patients, nasotracheal in one and cricothyroidotomy in one. the mean difficulty of intubation was $1.04 \pm 2.3$. The mean difficulty of auscultative confirmation was $0.92 \pm 1.4$. Twelve of 25 patients had witnessed cardiac arrests with a mean duration without perfusion of 13 minutes, the longest known duration being 40 minutes. The $\mathrm{CO}_{2}$ detector registered positive in all of these patients. In three of these patient, the detector initially registered negative then turned positive upon reintubation. Radiographic or visual documentation of incorrect placement was not obtained in these patients. Of the five unwitnessed cardiac arrests, $\mathrm{CO}_{2}$ was detected in three and was not detected in two. The remaining eight patients had respiratory failure and sustained some degree of perfusion. All had $\mathrm{CO}_{2}$ detected on the monitor. Comparison of 25 randomly selected controls revealed no statistically significant differences. One of these control patients had an esophageal intubation detected by visualization in the emergency department.

Conclusion: Based on preliminary data, the MSA Minicap II end-tidal, $\mathrm{CO}_{2}$ detector appears to be a rapid and accurate method of confirming endotracheal tube placement in the prehospital patient.
Track II

Great Hall Center
Friday, June 14, 1991

Moderator: Daniel Spaite

\author{
4:30 PM \\ Comparison of Albuterol Delivered by Nebulizer or Metered \\ Dose Inhaler with Spacer for the Treatment of Acute Asthma \\ Idris AH, McDermott MF, Morrabel A, RaucciJ \\ Division of Emergency Medicine, University of Florida College of \\ Medicine, Gainesville, Florida; Department of Emergency Medicine, \\ Cook County Hospital, Chicago, Illinois; University of Illinois College \\ of Medicine, Chicago, Ilinois
}

Hypothesis: Patients treated for acute asthma respond equally well to nebulized albuterol or to albuterol dose inhaler with a spacer device (MDIS).

Methods: Thirty patients with acute asthma were investigated in a prospective, randomized, double-blinded, multi-center study. Data were analyzed with two sample $t$-test, significance of $\mathrm{p}<.05$. Patients were treated every 30 minutes with four puffs of placebo MDIS followed by nebulized albuterol $2.5 \mathrm{mg}$ in $2 \mathrm{ml}$ of normal saline or with MDIS albuterol four puffs followed by placebo nebulizer ( $2.5 \mathrm{ml}$ normal saline). Spirometry followed each treatment.

Results: Patients had moderate to severe asthma. Before treatment fo the nebulizer and MDIS groups, respectively, the mean $( \pm \mathrm{SD})$ percentage forced expiratory volume in 1 second (\% $\mathrm{PFEV}^{1}$ ) was $37.2 \pm 19.1$ and $37.6 \pm 16.2$ (NS, $\mathrm{p}=.72$ ). After the first treatment, the mean \%PFEV1 increased by $16.7 \pm 11.1$ and $12.8 \pm 5.5(\mathrm{NS}, \mathrm{p}=.21$; power $=.91,10 \%$ difference of means), the mean maximum increase in \%PFEV1 was $25.8 \pm 11.7$ and $24.0 \pm 9.7$ (NS, $p=.66$; power $=.96,15 \%$ difference of means), and the mean duration of treatment was $125.0 \pm 82.0 \mathrm{~min}$ and 116.7 $\pm 73.0 \mathrm{~min}(\mathrm{NS}, \mathrm{p}=.78)$. Twenty-eight $(93 \%)$ patients were treated successfully within the study and discharged home.

Conclusion: Albuterol delivered by metered dose inhaler with a spacer device is an effective alternative for treating patients with moderate to severe asthma and may be useful for the prehospital treatment of asthma. 


\section{Track II}

Great Hall Center
Friday, June 14, 1991

Moderator: Daniel Spaite

\section{4:45 PM}

High Flow Sheath for Fluid Resuscitation:

A Comparison of Flow Rates with Standard Catheters

Idris $A H$, Melker RJ

Division of Emergency Medicine, University of Florida College of

Medicine, Gainesville, Florida; Department of Emergency Medicine,

Cook County Hospital, Chicago, Illinois; University of Illinois College

of Medicine, Chicago, Ilinois

Study objective: To study the flow rates of vascular infusion devices used in fluid resuscitation.

Methods: The fluid flow rates of 22, 20, 18, 16, and 14-g catheters, 4.0, 5.0, 6.0, and 8.5 French sheaths were measured. A 1liter plastic bag of Ringer's lactate was connected to a high-flow trauma fluid administration set which in turn was connected to one of the devices. The bottom of the bag was suspended one meter above the distal end of each device and the fluid was allowed to flow by gravity. Three units of each device were tested and the elapsed times of the three trials were averaged. The arithmetic means and standard deviation (SD) were then compared for the different devices. Significance testing was done using one-way analysis of variance and Duncan's multiple range test.

Results: The mean times ( $\pm \mathrm{SD}$ ) necessary to drain 1 liter of fluid ranged from $96 \pm 0.72$ seconds for the 8.5 French sheath to $1628+29.22$ seconds for the 22 gauge catheter. When compared to a 14-g catheter, the 8.5 French sheath flow rate was significantly faster $(\mathrm{p}<.0001)$.

Conclusion: The 8.5 and 6.0 French sheaths provided flow rates sufficient to treat severe volume depletion or blood loss in adults. The 14 and 16 gauge catheters and the 4.0 and 5.0 French sheaths had flow rates capable of treating severe pediatric hypovolemia.

Track III

Great Hall East/West

Friday, June 14, 1991

Moderator: Eric Davis

\author{
10:00 AM \\ To Report or Not to Report: Emergency \\ Services Response to. Elder Abuse \\ Walker G, Jones JS, Krohmer J \\ Emergency Medicine Residency Program, Butterworth Hospital, \\ Michigan State University College of Human Medicine, Grand \\ Rapids, Michigan
}

Purpose: Prehospital EMS personnel, as initial responders to calls for.assistance, are in an ideal position to identify abused or neglected elderly. A survey was conducted of prehospital personnel in Michigan to determine the scope of this problem, levels of awareness, and willingness to report cases of elder abuse.

Methods: The study population was a random sample of 500 prehospital personnel throughout one state. A blinded, selfadministered survey was completed by EMTs and paramedics outlining their training, demographic characteristics, and attitudes. Attitudes concerning the understanding and reporting of geriatric abuse were measured using a Likert scale.

Results: A total of 126 surveys (25\%) were completed; $70 \%$ of the respondents were paramedics. Respondents had an average of 7.7 years of prehospital emergency-care experience and evaluated 11 patients $>65$ years old each week. Seventy-six percent had seen a suspected case of elder abuse or neglect during their career; $68 \%$ had seen a case during the past 12 months (mean 2.98 cases/yr). Despite these numbers, surveyed personnel reported only $25 \%$ of suspected cases to authorities last year (mean 0.74 cases/yr). Reasons given for not reporting included: lack of standardized abuse protocols, unaware of mandatory reporting laws, limited knowledge of available services, and unclear definitions of abuse/neglect. Eighty-four percent of respondents stated that training related to elder abuse was not available through their EMS agency.

Conclusion: Paramedics and EMTs lack a complete understanding of their role in the identification and reporting of elder abuse. This information should be emphasized during EMS training and reinforced through continuing education. 


\section{Track III}

Great Hall East/West
Friday, June 14, 1991

Moderator: Eric Davis

\section{0:15 AM}

Analysis of Non-Productive Use of 911 Emergency Ambulance Resources: Implication for Policy and Research Taliaferro E, Saunders CE

University of California, San Francisco, School of Medicine;

Department of Public Health, City and County of San Francisco, Califormia

Purpose: To analyze the non-productive use of 911 emergency ambulance resources to determine what policy changes were suggested that would conserve EMS resources.

Methods: A retrospective analysis was performed on all nonproductive 911 ambulance runs in San Francisco over a onemonth period Non-productive runs are defined as those not resulting in patient treatment or transport). The appropriateness of non-transport was determined by a physician audit of those cases in which a transport decision was possible. The cost of the diversion of system resources was calculated using financial data, and the impact on the system response times was determined using a computer simulation model of the EMS system.

Results: of 5,632 runs dispatched, 2,202 (39\%) were non-productive. Patient contact was made in 1,040 (47\%) cases. of 702 cases in which a transport decision was made, $605(86 \%)$ were deemed appropriate, 84 (14\%) lacked adequate documentation to establish appropriateness, and $13(2 \%)$ were deemed inappropriate and should have been transported. The total annual cost to the city of San Francisco of non-productive runs was $\$ 3,550,099$, or $\$ 148$ per run. When non-productive runs were excluded from the EMS system by computer simulation, ambulance utilization fell $15.6 \%$ and response times for highpriority calls ( $45 \%$ of all calls) fell by 1.13 minutes.

Conclusion: Non-productive ambulance runs consume large amounts of EMS resources and lengthen response times for those truly in need. When patient contact is made, only rarely is the non-transport decision in error. Future research is needed to prospectively identify calls at dispatch which could safely utilize alternative resources or assistance.

Track III

Friday, June 14, 1991

Great Hall East/West
10:30 AM

Outcome of Patients Not Transported by Ambulance

Bryan ED, Zachariah BS, Griffin MP

University of Texas Medical School at Houston; Department of Surgery, Division of Emergency Medicine; Harris County Emergency Corps, Houston, Texas

Purpose: Few authors have addressed the medical and legal risk of ambulance patients (PTs) refusing or being refused transport, but no data exist on PT outcomes. A study was undertaken to determine why PTs were not transported and their subsequent outcomes.

Setting: A busy, urban, volunteer EMS system with indirect medical control.

Methods: This study involved the review of 158 consecutive charts where an ambulance was called but the PT was not transported (no-loads). The PT was contacted by telephone by the investigators. Telephone follow-up was available for 93 PTs $(59 \%)$. This follow-up was used to determine the PTs' outcome. The PTs also were asked why they were not transported. Results: of the $93 \mathrm{Pts}, 60$ went to a physician by another method. Of these $60,15(25 \%)$ were admitted, mean stay duration 6.6 days, median 3.0 days, range $1-30$ days. There were two ICU admissions and two deaths. Of the 93 respondents, 43 (46\%) were true PT refusals. Paramedics suggested no transport or agreed to no transport in 50 cases $(54 \%)$. These accounted for 11 of 15 hospitalizations $((73 \%)$. While not statistically significant, the trend was toward the paramedic-selected, no-loads to be more often admitted ( $22 \%$ vs $9 \%, p=.156$ Fisher's exact test).

Conclusions: A significant percentage of no-loads have serious, even fatal illnesses or injuries. When the decision not to load is made or supported by the paramedic, the $P T$ is twice as likely to require hospitalization, The data suggest that paramedics should always encourage patients to be transported, but further prospective study is needed. 
Track III

Great Hall East/West

Friday, June 14, 1991

Moderator: Eric Davis

\author{
10:45 AM \\ Unexpected ALS Procedures on Non-Emergency \\ Ambulance Calls: The Value of a Single-Tier System \\ Wilson BD, Gratton MC, Overton J, Watson W \\ Department of Emergency Medicine, University of Missouri, \\ Truman Medical Center West, Kansas City, Missouri
}

Objectives: For many years, debate has existed as to the best EMS system-single-tier or 2-tier providers. In a 2-tier system, a BLS unit provides triage when it responds to non-emergency (Code 3) calls. If on arrival an emergency is found, an ALS unit is called in. If unexpected ALS care was needed for calls dispatched as "non-emergency," this would be a weakness of 2-tier systems. The purpose of this study was to determine the frequency rate of ALS care in patients that underwent triage who were not predicted as Code 3 in a single-tier system, to estimate the problems with a 2-tier system.

Design: A single-tier, all ALS provider EMS system, serving an urban population of 450,000 with an annual response volume of 45,000 calls.

Participants: All Code 3 calls over a 12-month period were evaluated to determine the prevalence of unpredicted ALS care, defined as EKG monitoring, initiating or attempting IV access, administration of medications, or endotracheal intubation.

Results: A total of 6,362 Code 3 calls were identified; 309 were upgraded to emergency en route based on additional information obtained by dispatchers. Ff 6,053 Code 3 calls, 710 $(11.7 \%)$ involved the provision of one or more ALS procedures, with $296(4.9 \%)$ receiving an IV, and three requiring endotracheal intubation. The documentation rate of procedures on run tickets reviewed was $98 \%$, compared to computerized data. Calls upgraded by dispatchers required one or more procedure in $144 / 309$ calls $(46.6 \%)$ more frequently than Code 3 calls $(\mathrm{p}<0.005)$.

Conclusion: Procedure rates for upgraded calls document that dispatchers properly establish call priority. Despite strict dispatching protocols, $11.7 \%$ of Code 3 calls required ALS procedures. If these results are similar in 2-tier systems, this supports the need for all ALS, single-tier systems.
Track III

Great Hall East/West
Friday, June 14, 1991

Moderator: Eric Davis

\author{
11:00 AM \\ Analysis of Ambulance "Response Time" and Component \\ Intervals: Suggestions for Standard Definitions \\ Saunders CE, Amick P, ApplegarthI \\ University of Califormia, San Francisco; Department of Health, \\ City and County of San Francisco, Califormia
}

Purpose: "Response Time" is a key variable in evaluating cardiac arrest survival and for inter-system comparisons, yet definitions vary widely. This study sought to identify and define common functional components and devise a standard definition.

Methods: This study surveyed medium-sized cities in the United States to identify and define common functional components and devise a standard definition. A retrospective study of ambulance response time intervals also was conducted for all critical calls during a one-month period in San Francisco based on dispatch data.

Results: Of 30 cities surveyed, $24(80 \%)$ responded, and common functional component time intervals were identified. Using these definitions, mean intervals for San Francisco were calculated $(n=1,519)$; initial receipt at 911 answering point (PSAP) to transfer to medical dispatcher (PASP-I, $0.52 \mathrm{~min}$ ), arrival at medical communications center to dispatch queue (MEDCOM-I, $0.65 \mathrm{~min}$ ), dispatch queue to ambulance assignment (QUEUE-I, $2.08 \mathrm{~min}$ ), ambulance assignment to arrival at scene (OUTBOUND-I, $5.28 \mathrm{~min}$ ), arrival at scene to departure from scene (SCENE-I, $17.61 \mathrm{~min}$ ), departure from scene to arrival at the destination (TRANSPORT-I, $12.94 \mathrm{~min}$ ), arrival at destination to back in service (RECOVERY-I, $25.41 \mathrm{~min}$ ), initial receipt at PASP to arrival at scene (TOTRESP-I, $8.53 \mathrm{~min}$ ), arrival at medical communications center to arrival at scene (MEDRESP-I, $8.01 \mathrm{~min}$ ).

Conclusions: The time intervals identified represent different functional components of an EMS system and have defined end points. This study recommends that TOTRESP-I be used in cardiac arrest studies and for inter-system comparisons. Intervals should be separately determined for first responder versus advanced life support (ALS) ambulance arrival. 
Track III

Friday, June 14, 1991

Great Hall East/West
Track III

Great Hall East/West
Friday, June 14, 1991

Moderator: Eric Davis

\section{1:30 AM \\ EMS Quality Management: Implementing an \\ Operationally Integrated Quality Control Program \\ Saunders $C E$ \\ University of California, San Francisco School of Medicine, and \\ Department of Public Health, City and County of San Francisco}

Purpose: Industrial Concepts of Quality Control (QC) differ from traditional medical Quality Assurance (QU) by their focus on managing quality through operationally integrated, closedloop, control systems, and real-time control processes. The QA programs in emergency medical services (EMS) usually lack this degree of direct operational integration, and control processes often have a long lag time and exert controls only weakly or indirectly. this study reports the feasibility and results of a broad-based QC system implementation in San Francisco.

Methods: A QC system was implemented which had the following components: defined clinical and operational performance variables and set standards; computer-based performance monitoring; and multiple, closed-loop, real-time, operationally integrated, control pathways (feedback, feed-forward, and steering), Specially trained, paramedic quality control officers and fulltime physician medical direction was integrated into the operating system. Performance data and quality control management activity data were collected prospectively (105 variables).

Results: In the first 12 months, over 200,000 data elements have been collected and analyzed, 52 separate reports are regularly prepared and utilized operationally, and 7,000 hours of inservice instruction have been provided to paramedics, including 32 new protocols. In-field quality control personnel have responded to and corrected 96 clinical complaints or unusual occurrences and made 1,812 real-time, field contacts or process adjustments.

Conclusions: The QC programs are feasible in the EMS industry. Studies are underway to determine this program's effectiveness using outcome measures.

\section{1:45}

The Prospective Analysis of the Impact of the Number of Helicopters on Emergency Air Medical Transport in a Regional EMS System

Brophy T, Spaite DW, Valenzuela TD, Meislin $H W$,

Criss EA, Hinsberg $P$

Arizona Emergency Medicine Research Center, University of Arizona, College of Medicine, Tucson, Arizona

Introduction: Changing the number or helicopters available to an EMS system alters the use patterns of air medical transport. Methods: Prospective evaluation of helicopter dispatch data from a centralized EMS dispatch agency in a regional EMS system. During the study period, four alterations in the total number of helicopters available to the system occurred $(1,2,3,2,3)$. Statistical analysis consisted of Chi Square with Yates' correction and comparison of sample proportions with $\mathrm{p}<.05$ considered significant.

Results: A total of 667 helicopter dispatches occurred during the 20 -month study $(4 / 89-11 / 90)$.

\begin{tabular}{|c|c|c|}
\hline \multicolumn{3}{|c|}{$\begin{array}{c}\text { Helicopter Dispatches Days of Patients Cancellation } \\
\text { Available Per Dav }\end{array}$} \\
\hline 1 & $113 / 170=\overline{0.66}$ & $67 / 170=39 \%$ \\
\hline 2 & $165 / 171=0.96$ & $85 / 171=50 \%$ \\
\hline 3 & $389 / 259=1.50$ & $193 / 259=74 \%$ \\
\hline Total & $667 / 600=1.11$ & $345 / 600=58 \%$ \\
\hline p-valu & $<.01$ & $<.01$ \\
\hline
\end{tabular}

\begin{tabular}{lrr} 
& \multicolumn{2}{c}{ Utilization Per Day Rate } \\
\cline { 2 - 2 } 1 & $\mathbf{5 1 7 0}=0.30$ & $62 / \mathbf{1 1 3}=\mathbf{5 5} \%$ \\
2 & $73 / 171=0.43$ & $92 / 165=\mathbf{5 6} \%$ \\
3 & $192 / 259=0.74$ & $197 / \mathbf{3 8 9}=\mathbf{5 1 \%}$ \\
Total & $316 / 600=0.53$ & $351 / 667=53 \%$ \\
p-value & $<.01$ & .474
\end{tabular}

Conclusions: Changes in dispatch patterns could result either from increased availability or alterations in the dispatchers' "threshold" for use based upon a perceived lessening of the need to save a "scarce" resource. Had the second possibility played a significant role, the rate of cancellation by ground personnel after arrival at the scene would be expected to have increased. Since this did not occur, it is likely that the increased use was actually a result of increased availability. In systems that dispatch helicopters prior to ground personnel arrival, evaluation of cancellation rates may provide useful information for analyzing the impact of system alterations. 